\title{
Ictiofauna capturada incidentalmente en la pesquería de langostino patagónico Pleoticus muelleri Bate, 1888
}

\author{
Fish bycatch in the Patagonian shrimp fishery Pleoticus muelleri Bate, 1888 \\ María E. Góngora ${ }^{1,3}$, Nelson D. Bovcon ${ }^{2,3}$ y Pablo D. Cochia ${ }^{3}$ \\ ${ }^{1}$ Secretaría de Pesca de la Provincia del Chubut. Belgrano 778, $1^{\text {er }}$ piso. Rawson, Chubut, Argentina, CP 9103 \\ ${ }^{2}$ Consejo Nacional de Investigaciones Científicas y Técnicas (CONICET), Argentina \\ ${ }^{3}$ Facultad de Ciencias Naturales de la Universidad Nacional de la Patagonia, \\ Sede Trelew. Julio A. Roca 115, $1^{\text {er }}$ piso. Trelew, Chubut, Argentina, CP 9100 \\ mariaevagongora@hotmail.com
}

\begin{abstract}
The catch of non-target animal species, known as bycatch, and their discard are main problems in fisheries management. The prawn or shrimp trawl fisheries in particular are recognized for their incidence in the global discards. In Argentine Patagonia there is an industrial shrimp fishery based on the species Pleoticus muelleri, of high commercial value. Since 2001 an on-board observer program collects information about all species caught. We analyzed the composition of the bycatch of fishes in the Patagonian shrimp fishery and the frequency of species occurrence. The data analyzed correspond to the outrigger freezing fleet, which catches more than $75 \%$ of the total shrimp landings in Argentina. We identified 67 species of osteichthyes, chondrichthyes and myxini, corresponding to 42 families. The families with the larger number of species were Rajidae (9), Clupeidae (3), Ophidiidae (3), Zoarcidae (3), Nototheniidae (3) y Paralichthyidae (3). Of the 67 species reported as bycatch, seven had not been reported before in the San Jorge gulf and adjacent waters: Atlantoraja cyclophora, Brevoortia aurea, Diplodus argenteus, Pagrus pagrus, Micropogonias furnieri, Sarda sarda and Paralichthys patagonicus.
\end{abstract}

Key words: Solenoceridae, non-target species, San Jorge Gulf, ecosystem-based management, Argentina

\section{Introducción}

El efecto de la pesca en los ecosistemas marinos ha recibido una atención creciente en los últimos años. Uno de los impactos directos más visibles es la captura de especies animales no objetivo o captura incidental (Kelleher 2008). En las pesquerías de arrastre de camarón y langostino, la mayoría de las especies que componen la captura incidental son descartadas al mar (Stobuzki et al. 2001). A nivel mundial, las estimaciones sobre el descarte señalaron que estas pesquerías, y en particular las pesquerías camaroneras tropicales, fueron por sí solas
Resumen.- La captura de especies animales no objetivo y su posterior descarte al mar son problemas centrales en el manejo pesquero. Las pesquerías de langostino y camarón, en particular, son reconocidas por su alta incidencia en el descarte mundial. En aguas de la Patagonia argentina se desarrolla una pesquería industrial de langostino sustentada por la especie Pleoticus muelleri, de alto valor económico. Desde el año 2001 un programa de observadores a bordo recolecta información no sólo de la especie objetivo de la pesquería sino de todas las especies capturadas. En este trabajo analizamos la composición y frecuencia de ocurrencia de la ictiofauna capturada incidentalmente en la pesquería del langostino patagónico. Los datos analizados corresponden a la flota congeladora arrastrera que opera con dos redes langostineras, una por banda, llamada flota congeladora tangonera, la que captura más del $75 \%$ de los desembarcos de langostino en Argentina. Se identificaron 67 taxones de peces óseos, cartilaginoso y mixines, correspondientes a 42 familias. Las familias con mayor número de especies presentes fueron Rajidae (9), Clupeidae (3), Ophidiidae (3), Zoarcidae (3), Nototheniidae (3) y Paralichthyidae (3). De los 67 taxones registrados, siete de ellos no estaban citados para el golfo San Jorge y aguas adyacentes: Atlantoraja cyclophora, Brevoortia aurea, Diplodus argenteus, Pagrus pagrus, Micropogonias furnieri, Sarda sarda y Paralichthys patagonicus.

Palabras clave: Solenoceridae, captura incidental, golfo San Jorge, manejo ecosistémico, Argentina responsables de más del 27\% (1,86 millones de toneladas) del total de los descartes estimados para el período 19942003. La tasa de descarte para todas las pesquerías de arrastre de langostino y camarón fue de $62,3 \%$ para el mismo periodo (excluyendo las pesquerías chinas) (Kelleher 2008).

La preocupación por las elevadas capturas de especies no objetivo en las pesquerías de langostino y camarón está focalizada en especies que son objetivo de otras pesquerías comerciales o recreacionales, o en especies en peligro o vulnerables (Stobutzki et al. 2001). Sin 


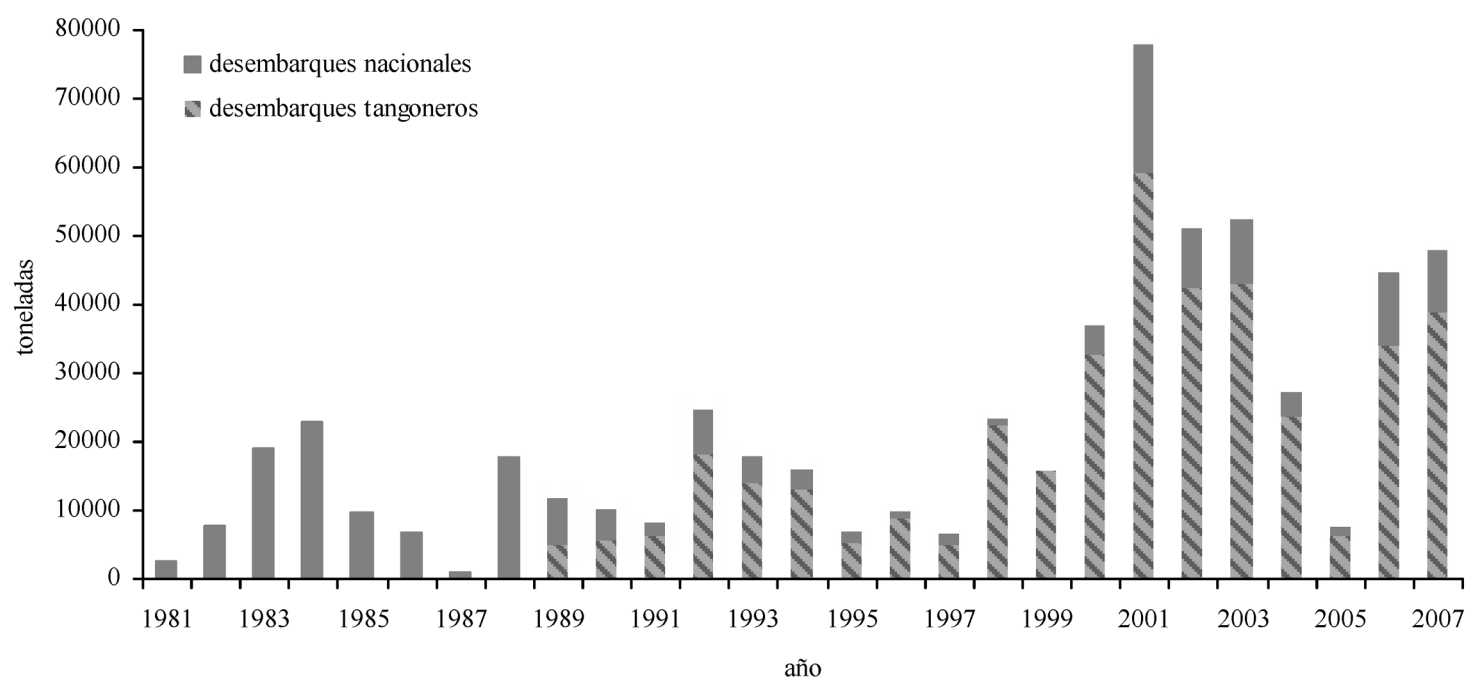

Figura 1

Desembarques de langostino patagónico en Argentina. Se indican los totales y los correspondientes a la flota congeladora tangonera entre 1981 y 2007

Patagonian shrimp landings in Argentina. Total and outrigger freezing fleet landings between 1981 and 2007 are indicated

embargo, un número significativo de especies no comerciales son también capturadas incidentalmente. Si se adopta un enfoque de ecosistemas en la pesca (FAO 2003), la sustentabilidad de todas las especies que componen la captura incidental debe ser atendida (Stobustzki et al. 2001).

En aguas de la Patagonia argentina se desarrolla una pesquería industrial de langostino sustentada por la especie Pleoticus muelleri Bate, 1888, de alto valor económico. Las capturas de esta especie han sido muy irregulares (Fig. 1). Desde mediados de los años ochenta, cuando se detectaron concentraciones importantes de langostino en la zona del golfo San Jorge (Fig. 2), hasta fines de los noventa, las capturas se mantuvieron por debajo de las $25.000 \mathrm{t}$. A partir del año 2000 las capturas aumentaron llegando a un record histórico de 78.000 t en 2001 y permanecieron por encima de las 27.000 t hasta el presente, con excepción del año 2005, cuando la captura descendió a 7.400 t.
Actualmente en la pesquería de langostino patagónico participan entre 70 y 80 barcos congeladores arrastreros que operan con dos redes langostineras, una por banda, llamados barcos congeladores tangoneros, los que ingresaron a fines de los años ochenta y principios de los noventa. A partir de 1991 , más del $75 \%$ de los desembarques de langostino nacionales provinieron de la flota congeladora tangonera (Fig. 1).

La pesquería se desarrolla en aguas de jurisdicción provincial (provincias de Chubut y Santa Cruz), en el golfo San Jorge y zonas costeras, y en aguas de jurisdicción nacional, en lo que en la actualidad es un área de veda decretada para la protección de juveniles de merluza (Merluccius hubssi) ${ }^{1}$ (Fig. 2).

La captura incidental de merluza es el problema más serio que enfrenta el manejo de la pesquería de langostino patagónico. La tasa merluza / langostino en esta pesquería varió entre 0,46 y 1,44 en el período 1998-2004 (Cordo $2005^{2}$ ). La merluza es el principal recurso de las flotas

${ }^{1}$ En la Argentina, el mar territorial fue cedido a las provincias con costa marítimas, y desde las 12 a las 200 millas sobre la plataforma continental la jurisdicción la ejerce el gobierno de la Nación Argentina (Ley Federal de Pesca 24.922).

${ }^{2}$ Cordo H. 2005. Evaluación del estado del efectivo sur de $41^{\circ} \mathrm{S}$ de la merluza (Merluccius hubbsi) y estimación de la captura biológicamente aceptable correspondiente al año 2005. Informes Técnicos Oficiales $\mathrm{N}^{\circ}$ 37. 23-05-05. Instituto de Investigación y Desarrollo Pesquero, Mar del Plata, Argentina, 29 pp. [resumen] <http://www.inidep.edu.ar/informes/tec/05.htm〉 


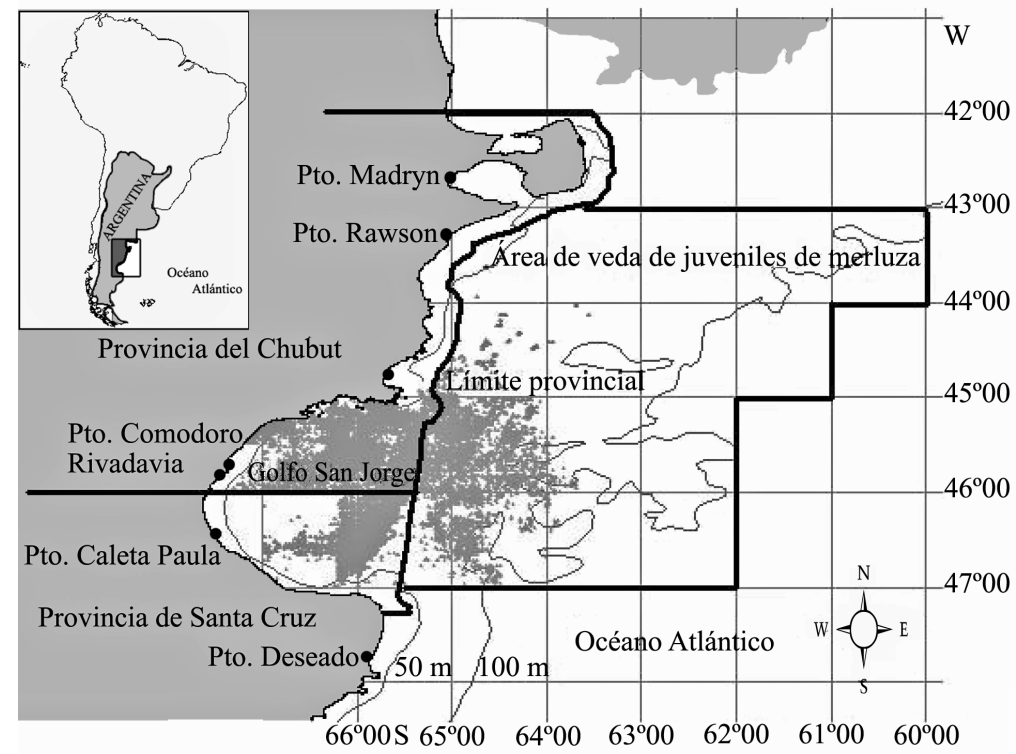

Figura 2

Áreas de operación de la pesquería de langostino patagónico y lances observados en la flota congeladora tangonera, desde 2003 a 2007. Cada punto gris corresponde a un lance de pesca observado

Patagonian shrimp fishing grounds and observed hauls on outrigger freezing fleet from 2003 to 2007. Gray dots show the location of each observed haul

fresqueras del país y su pesquería está actualmente en crisis (Cordo $2006^{3}$ ). La pesquería de langostino se desarrolla en dos áreas de cría de merluza: el golfo San Jorge y el área de veda de juveniles de merluza (Bezzi et al. 2004, Aubone et al. 2004). Las medidas de manejo tomadas en la pesquería de langostino patagónico estuvieron focalizadas en la especie objetivo y en la captura incidental de merluza.

Desde el año 2001 la pesquería es monitoreada por el Programa de Observadores a Bordo perteneciente a la Provincia del Chubut. Con el objetivo de avanzar en un enfoque de ecosistemas en las pesquerías, el programa obtiene información no sólo de la especie objetivo sino de todas las especies capturadas.

El objetivo del presente trabajo fue analizar la ictiofauna capturada incidentalmente ${ }^{4}$ en la pesquería de langostino patagónico en la flota congeladora tangonera que opera en Patagonia argentina.

\section{Material y métodos}

Para el presente análisis se utilizó la información recolectada en la flota congeladora tangonera por el Programa de Observadores a Bordo perteneciente a la Provincia del Chubut. Estos buques operan con dos redes arrastreras langostineras, una por cada banda. El tamaño de las redes utilizadas varió de acuerdo con la potencia del barco, entre 30 y $50 \mathrm{~m}$ de relinga superior con aberturas verticales que oscilaron entre $1,2 \mathrm{~m}$ y $1,5 \mathrm{~m}$ (Roux et al. 2007).

Los observadores a bordo registraron en cada lance de pesca las especies capturadas junto a la especie objetivo. De cada especie se consignó la abundancia en número en cuatro categorías y su destino. Las categorías utilizadas en la estimación de la abundancia fueron: dominante (la especie representa más del $50 \%$ de la captura en número, su presencia da el aspecto general de la captura), abundante (entre el 25 y el 50\%, se observa

${ }^{3}$ Cordo H. 2006. Evaluación del estado del efectivo sur de $41^{\circ} \mathrm{S}$ de la merluza (Merluccius hubbsi) y estimación de la captura biológicamente aceptable correspondiente al año 2006. Informes Técnicos Oficiales $N^{o}$ 34. 12-07-06. Instituto de Investigación y Desarrollo Pesquero, Mar del Plata, Argentina, 27 pp. [resumen]. <http://www.inidep.edu.ar/informes/tec/06.htm>

${ }^{4}$ El término captura incidental es utilizado en el presente trabajo como captura de especies animales no objetivo, según la traducción de Kelleher (2005) en referencia al término 'bycatch' (Kelleher 2008). 
con facilidad su presencia), común (entre el 5 y el $25 \%$, se observa al prestar atención y revolver las capturas) y rara (menos que el 5\%, pocos ejemplares). El destino de la especie puede ser: totalmente encajonada (encajonada), parcialmente encajonada (parcialmente encajonada) o totalmente arrojada al mar (descartada).

Un total de 18.718 lances fueron examinados entre los $44^{\circ} 00^{\prime} \mathrm{S}$ y los $47^{\circ} 00^{\prime} \mathrm{S}$, en profundidades desde 13 a $116 \mathrm{~m}$ (Tabla 1, Fig. 2).

Se listaron todas las especies de peces observadas durante los lances de pesca de los años 2003 a 2007. Para la nomenclatura científica se utilizó el catálogo en versión electrónica de Eschmeyer (2007). No fueron considerados los años 2001 y 2002 porque en esos años se inició la capacitación en la identificación de especies.

Se calculó la frecuencia de ocurrencia (FO) para cada especie:

$\mathrm{FO}=$ (lances donde la especie fue identificada / lances totales) 100

como un criterio para caracterizar el problema de la captura incidental (Hall 1996, Hall et al. 2000). Para el cálculo de la FO se utilizaron datos de los años 2003, 2004, 2006 y 2007. Se excluyó el año 2005 debido a las fuertes restricciones espaciales y temporales al esfuerzo de pesca decretadas en respuesta a la baja abundancia de langostino registrada ese año.

\section{Tabla 1}

\section{Número de lances examinados por mes y año del Programa de Observadores a Bordo de la Provincia del Chubut}

Number of hauls examined by month and year of Programme of Observers on Board of the Province of Chubut

\begin{tabular}{lrrrrrr}
\hline & 2003 & 2004 & 2005 & 2006 & 2007 & Total \\
\hline Ene & 131 & & & & & 131 \\
Feb & 144 & 216 & 1396 & 2 & & 1758 \\
Mar & 719 & 802 & 399 & & 844 & 2764 \\
Abr & 415 & 446 & 71 & 607 & 497 & 2036 \\
May & 456 & 567 & 89 & 747 & 404 & 2263 \\
Jun & 375 & 1096 & & 283 & 98 & 1852 \\
Jul & 466 & 555 & & 372 & 298 & 1691 \\
Ago & 682 & 781 & & 266 & 397 & 2126 \\
Sep & 486 & 446 & & 302 & 237 & 1471 \\
Oct & 266 & 325 & 234 & 194 & 237 & 1256 \\
Nov & 277 & 125 & 175 & & & 577 \\
Dic & 178 & & 615 & & & 793 \\
Total & 4595 & 5359 & 2979 & 2773 & 3012 & 18718 \\
\hline
\end{tabular}

Los rangos de frecuencia de ocurrencia por especie fueron construidos utilizando métodos de remuestreo con reemplazo (Crawley 2007).

Exceptuando el año 2005 por las razones antes mencionadas, se calculó la frecuencia en que la especie fue observada como rara, común, abundante o dominante:

$F_{\text {Rara }}=$ (lances donde la especie fue Rara / lances donde la especie fue identificada) 100

$F_{C o m i n}=$ (lances donde la especie fue Común / lances donde la especie fue identificada) 100

$F_{\text {Abundante }}=$ (lances donde la especie fue Abundante / lances donde la especie fue identificada) 100

$F_{\text {Dominate }}=$ (lances donde la especie fue Dominante / lances donde la especie fue identificada) 100

En los lances en los que la especie fue identificada, se calculó el porcentaje en que fue encajonada total o parcialmente:

$F_{\text {Encaionada }}=$ (lances donde la especie fue Encajonada / lances donde la especie fue identificada) 100

$F_{\text {Parcial.Encaj. }}=$ (lances donde la especie fue Parcialmente Encajonada / lances donde la especie fue identificada) 100

\section{Resultados}

La lista de especies registradas comprendió 44 peces óseos, 20 peces cartilaginosos y dos especies de mixines, correspondientes a 42 familias (Tabla 2). Las familias con mayor número de especies presentes fueron Rajidae (9), Ophidiidae (3), Zoarcidae (3), Nototheniidae (3) y Paralichthyidae (3).

Desde un enfoque zoogeográfico y siguiendo lo propuesto por Menni \& Gosztonyi (1982) se reconocen 13 especies del distrito Bonaerense, ocho especies del distrito Magallánico, nueve especies pertenecientes a la denominada fauna mixta de la plataforma interna, 13 especies de amplia distribución en el mar argentino, una especie de aguas profundas y cinco especies no agrupadas. Por otro lado, 17 especies no se encuentran citadas en el trabajo mencionado. De las 66 especies registradas, siete no se encuentran citadas hasta el presente para el golfo San Jorge y aguas adyacentes: Atlantoraja cyclophora, Brevoortia aurea, Diplodus argenteus, Pagrus pagrus, Micropogonias furnieri, Sarda sarda y Paralichthys patagonicus, todas ellas pertenecientes a aguas templado-calidas y varias de ellas pertenecientes al distrito Bonaerense (Tabla 3). 


\section{Tabla 2}

Lista taxonómica de especies de peces capturadas incidentalmente en la pesquería de langostino patagónico por la flota tangonera desde 2003 a 2007

Taxonomic list of fish species reported as bycatch in the Patagonia shrimp fishery (outrigger freezing fleet) from 2003 to 2007

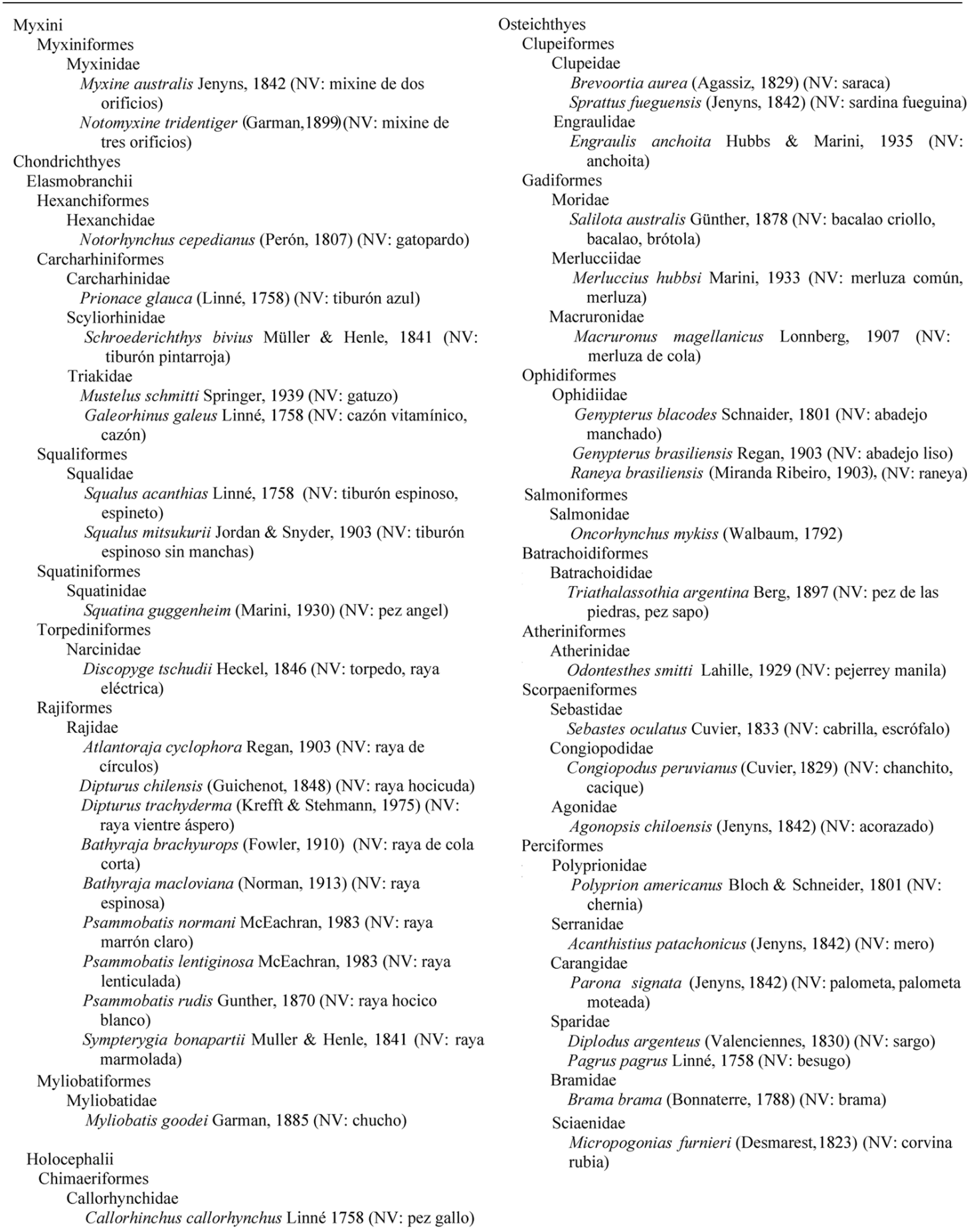


Tabla 3

Ubicación de las especies de peces registradas desde un enfoque zoogeográfico según esquema propuesto por Menni \& Gosztonyi (1982)

Position of fish species reported as bycatch in the Patagonia shrimp fishery within a zoogeographical context in accordance with Menni \& Gosztonyi (1982)

\begin{tabular}{|c|c|c|c|c|c|c|c|}
\hline & $\begin{array}{l}\text { Fauna del Distrito } \\
\text { Bonaerense }\end{array}$ & $\begin{array}{l}\text { Fauna del Distrito } \\
\text { Magallánico }\end{array}$ & $\begin{array}{l}\text { Fauna mixta de la } \\
\text { plataforma interna }\end{array}$ & $\begin{array}{l}\text { Fauna de amplia } \\
\text { distribución }\end{array}$ & $\begin{array}{c}\text { Fauna de } \\
\text { profundidad }\end{array}$ & $\begin{array}{c}\text { Especies no } \\
\text { agrupadas }\end{array}$ & Especies no citadas \\
\hline 1 & Mustelus schmitti & $\begin{array}{l}\text { Bathyraja } \\
\quad \text { macloviana }\end{array}$ & Galeorhinus galeus & $\begin{array}{l}\text { Schroederichthys } \\
\text { bivius }\end{array}$ & $\begin{array}{l}\text { Allocyttus } \\
\text { verrucosus }\end{array}$ & Myxine australis & $\begin{array}{l}\text { Notorhynchus } \\
\text { cepedianus }\end{array}$ \\
\hline 2 & $\begin{array}{l}\text { Squatina } \\
\quad \text { guggenheim }\end{array}$ & $\begin{array}{l}\text { Sprattus } \\
\quad \text { fueguensis }\end{array}$ & Squalus acanthias & Dipturus chilensis & & $\begin{array}{l}\text { Notomyxine } \\
\text { tridentiger }\end{array}$ & Prionace glauca \\
\hline 3 & $\begin{array}{r}\text { Myliobatis } \\
\text { goodei }\end{array}$ & Salilota australis & Squalus mitsukurii & $\begin{array}{l}\text { Bathyraja } \\
\quad \text { brachyurops }\end{array}$ & & $\begin{array}{l}\text { Dipturus } \\
\quad \text { trachyderma }\end{array}$ & $\begin{array}{l}\text { Psammobatis } \\
\text { lentiginosa }\end{array}$ \\
\hline 4 & $\begin{array}{l}\text { Engraulis } \\
\text { anchoita }\end{array}$ & $\begin{array}{l}\text { Macruronus } \\
\text { magellanicus }\end{array}$ & Discopyge tschudii & $\begin{array}{l}\text { Psammobatis } \\
\text { normani }\end{array}$ & & $\begin{array}{l}\text { Eleginops } \\
\text { maclovinus }\end{array}$ & Brevoortia aurea \\
\hline 5 & $\begin{array}{l}\text { Raneya } \\
\quad \text { brasiliensis }\end{array}$ & $\begin{array}{l}\text { Austrolycus } \\
\text { laticinctus }\end{array}$ & $\begin{array}{l}\text { Sympterygia } \\
\text { bonapartii }\end{array}$ & $\begin{array}{l}\text { Psammobatis } \\
\text { rudis }\end{array}$ & & Seriolella porosa & $\begin{array}{l}\text { Genypterus } \\
\text { brasiliensis }\end{array}$ \\
\hline 6 & Parona signata & $\begin{array}{l}\text { Illuocoetes } \\
\text { fimbriatus }\end{array}$ & $\begin{array}{l}\text { Callorhinchus } \\
\text { callorhynchus }\end{array}$ & Merluccius hubbsi & & & Odontesthes smitti \\
\hline 7 & Pagrus pagrus & Cottoperca gobio & $\begin{array}{l}\text { Polyprion } \\
\quad \text { americanus }\end{array}$ & $\begin{array}{l}\text { Genypterus } \\
\text { blacodes }\end{array}$ & & & $\begin{array}{l}\text { Agonopsis } \\
\quad \text { chiloensis }\end{array}$ \\
\hline 8 & $\begin{array}{l}\text { Micropogonias } \\
\text { furnieri }\end{array}$ & $\begin{array}{l}\text { Patagonotothen } \\
\text { ramsayi }\end{array}$ & $\begin{array}{l}\text { Acanthistius } \\
\text { patachonicus }\end{array}$ & Sebastes oculatus & & & $\begin{array}{l}\text { Diplodus } \\
\quad \text { argenteus }\end{array}$ \\
\hline 9 & $\begin{array}{c}\text { Nemadactylus } \\
\text { bergi }\end{array}$ & & $\begin{array}{l}\text { Pseudopercis } \\
\text { semifasciata }\end{array}$ & $\begin{array}{l}\text { Congiopodus } \\
\text { peruvianus }\end{array}$ & & & Brama brama \\
\hline 10 & $\begin{array}{l}\text { Percophis } \\
\quad \text { brasiliensis }\end{array}$ & & & $\begin{array}{l}\text { Stromateus } \\
\quad \text { brasiliensis }\end{array}$ & & & Dadyanus insignis \\
\hline 11 & $\begin{array}{l}\text { Pinguipes } \\
\text { brasilianus }\end{array}$ & & & $\begin{array}{l}\text { Paralichthys } \\
\text { patagonicus }\end{array}$ & & & $\begin{array}{c}\text { Paranotothenia } \\
\text { angustata }\end{array}$ \\
\hline 12 & $\begin{array}{l}\text { Atlantoraja } \\
\text { cyclophora }\end{array}$ & & & $\begin{array}{c}\text { Paralichthys } \\
\text { isosceles }\end{array}$ & & & Scomber japonicus \\
\hline 13 & $\begin{array}{c}\text { Triathalassothia } \\
\text { argentina }\end{array}$ & & & Xystreurys rasile & & & $\begin{array}{r}\text { Pomatomus } \\
\text { saltatrix }\end{array}$ \\
\hline 14 & & & & & & & Sarda sarda \\
\hline 15 & & & & & & & $\begin{array}{l}\text { Leptonotus } \\
\quad \text { blainvilleanus }\end{array}$ \\
\hline 16 & & & & & & & $\begin{array}{l}\text { Notothenia } \\
\quad \text { magellanica }\end{array}$ \\
\hline 17 & & & & & & & $\begin{array}{l}\text { Oncorhynchus } \\
\text { mykiss }\end{array}$ \\
\hline
\end{tabular}

peces registrado como captura incidental de esta pesquería en estudios previos (Pettovello 1999, Roux et al. 2007). Según Roux et al. (2007) los organismos que predominaron en la captura fueron principalmente equinodermos, moluscos, crustáceos y aproximadamente 30 especies de peces. En cuanto a la presencia de especies vulnerables, Cedrola et al. (2004) identificaron siete especies de rayas capturadas incidentalmente, mientras que en este trabajo se reportaron nueve especies de la familia Rajidae.

En las pesquerías de arrastre de langostino del norte de Australia y del estrecho de Torres se identificaron 350 especies de peces óseos y elasmobranquios. Las capturas estuvieron dominadas por un gran número de especies con baja abundancia (Stobustzki et al. 2001). Por el contrario, en la pesquería de langostino del sur y sudeste de Brasil fueron identificadas 91 especies (Vianna \& Almeida 2005), pero pocas de ellas contribuyeron significativamente a la biomasa y número total de individuos capturados. Ambas pesquerías se desarrollan en ecosistemas tropicales.

En la pesquería de langostino patagónico, la merluza fue la especie más importante en la ictiofauna capturada incidentalmente por la flota congeladora tangonera, tanto por su alta FO como por ser la especie que más contribuyó en número de individuos a la captura total. Para el período 1996-1997, Pettovello (1999) observó su presencia en el $91 \%$ de las muestras analizadas. 


\section{Tabla 4}

Frecuencia de ocurrencia por año de las especies de peces capturadas incidentalmente en la pesquería de langostino patagónico por la flota tangonera

Frequency of species bycatch occurrence by year in the Patagonia shrimp trawl fishery (outrigger freezing fleet)

\begin{tabular}{|c|c|c|c|c|c|c|c|c|c|c|}
\hline \multirow[b]{3}{*}{ Lances observados } & \multicolumn{2}{|c|}{2003} & \multicolumn{2}{|c|}{2004} & \multicolumn{2}{|r|}{2006} & \multicolumn{2}{|r|}{2007} & \multicolumn{2}{|c|}{$2003,2004,2006,2007$} \\
\hline & FO & Rango & FO & rango & FO & rango & FO & rango & FO & rango \\
\hline & & & & 359 & & 2773 & & 3012 & & 5739 \\
\hline Merluccius hubbsi & 99,2 & $98,7-99,7$ & 99,7 & $99,4-99,9$ & 99,57 & $99,1-100$ & 97,0 & $95,8-98,2$ & 99,04 & $98,7-99,3$ \\
\hline Patagonotothen ramsayi & 66,8 & $64,2-69,7$ & 83,0 & $81,1-84,9$ & 77,7 & $74,6-81,2$ & 65,9 & $62,1-69,0$ & 74,09 & $72,8-75,4$ \\
\hline Genypterus blacodes & 81,4 & $79,2-83,6$ & 84,4 & $82,5-86,4$ & 53,9 & $50,0-57,5$ & 57,8 & $54,3-61,3$ & 73,08 & $71,7-74,3$ \\
\hline Dipturus chilensis & 62,5 & $60,0-65,0$ & 76,5 & $74,3-79,0$ & 49,2 & $45,7-52,3$ & 57,4 & $53,8-60,7$ & 63,93 & $62,4-65,5$ \\
\hline Psammobatis normani & 67,4 & $64,5-70,3$ & 74,8 & $72,4-77,3$ & 26,1 & $23,0-29,8$ & 37,3 & $34,3-41,0$ & 56,89 & $55,5-58,4$ \\
\hline Discopyge tschudii & 64,8 & $62,1-67,3$ & 33,5 & $31,1-35,8$ & 44,3 & $41,1-47,7$ & 58,0 & $54,0-61,5$ & 49,22 & $47,8-50,9$ \\
\hline Stromateus brasiliensis & 40,5 & $37,9-43,5$ & 50,5 & $47,6-52,9$ & 32,9 & $29,7-36,2$ & 40,4 & $37,2-44,3$ & 42,53 & $41,2-43,9$ \\
\hline Sympterygia bonapartii & 27,1 & $24,6-29,7$ & 36,3 & $33,7-38,7$ & 32,1 & $28,4-35,4$ & 54,2 & $50,7-57,8$ & 36,29 & $34,7-37,8$ \\
\hline Schroederichthys bivius & 26,7 & $24,5-29,2$ & 24,4 & $22,2-26,5$ & 23,4 & $20,2-26,7$ & 14,7 & $12,2-17,1$ & 23,03 & $21,8-24,4$ \\
\hline Squalus acanthias & 27,6 & $25,0-30,3$ & 17,4 & $15,4-19,4$ & 22,4 & $19,4-25,6$ & 18,4 & $15,5-21,3$ & 21,44 & $20,3-22,8$ \\
\hline Congiopodus peruvianus & 24,9 & $22,2-27,6$ & 14,4 & $12,7-16,2$ & 16,6 & $13,6-18,9$ & 24,4 & $21,8-27,8$ & 19,77 & $18,6-20,9$ \\
\hline Callorhinchus callorhynchus & 14,1 & $12,1-16,0$ & 17,6 & $15,7-19,6$ & 16,6 & $14,1-19,6$ & 21,9 & $18,9-25,1$ & 17,23 & $16,1-18,4$ \\
\hline Xystreurys rasile & 0,6 & $0,2-1,10$ & & & 32,0 & $28,8-35,6$ & 48,5 & $45,4-52,1$ & 15,10 & $14,1-16,2$ \\
\hline Paralichtys isosceles & 10,3 & $8,4-12,1$ & 2,6 & $1,7-3,6$ & 26,9 & $23,4-30,55$ & 24,2 & $21,2-27,3$ & 13,27 & $12,3-14,3$ \\
\hline Cottoperca gobio & 11,1 & $9,4-13,0$ & 17,7 & $15,7-19,7$ & 3,6 & $2,3-5,0$ & 15,5 & $13,1-17,7$ & 12,87 & $11,9-13,9$ \\
\hline Raneya brasiliensis & 8,5 & $7,0-10,0$ & 2,2 & $1,4-3,0$ & 21,5 & $18,8-24,2$ & 27,5 & $24,5-31,1$ & 12,28 & $11,4-13,3$ \\
\hline Nemadactylus bergi & 16,6 & $14,7-19,0$ & 4,7 & $3,6-5,8$ & 7,1 & $5,3-8,9$ & 22,9 & $19,9-25,9$ & 12,07 & $11,1-13,1$ \\
\hline Genypterus brasiliensis & 9,9 & $8,2-11,6$ & 10,0 & $8,6-11,6$ & 10,0 & $8,0-12,3$ & 9,6 & $7,5-12,0$ & 9,91 & $9-10,8$ \\
\hline Sebastes oculatus & 7,8 & $6,2-9,3$ & 7,5 & $6,1-9,0$ & 8,0 & $5,9-10,8$ & 8,4 & $6,34-10,7$ & 7,85 & $6,9-8,6$ \\
\hline Mustelus schmitti & 16,0 & $14,1-18,3$ & 1,5 & $0,9-2,2$ & 2,1 & $1,1-3,2$ & 11,4 & $9,4-13,6$ & 7,73 & $6,9-8,5$ \\
\hline Percophis brasiliensis & 7,2 & $5,8-8,7$ & 4,8 & $3,7-6,0$ & 6,6 & $4,8-8,6$ & 14,5 & $12,0-17,0$ & 7,67 & $6,8-8,7$ \\
\hline Salilota australis & 4,4 & $3,2-5,6$ & 5,1 & $4,1-6,2$ & 18,8 & $16,2-21,6$ & 3,5 & $2,3-5,1$ & 6,98 & $6,2-7,7$ \\
\hline Parona signata & 8,2 & $6,6-9,7$ & 4,9 & $3,8-6,0$ & 5,2 & $3,6-6,9$ & 9,9 & $7,6-12,2$ & 6,89 & $5,9-7,7$ \\
\hline Pseudopercis semifasciata & 6,5 & $5,2-8,0$ & 1,8 & $1,1-2,6$ & 3,2 & $1,9-4,7$ & 12,2 & $10,0-14,6$ & 5,42 & $4,8-6,2$ \\
\hline Galeorhinus galeus & 12,4 & $10,6-14,2$ & 1,9 & $1,2-2,7$ & 3,0 & $1,9-4,3$ & 1,2 & $0,6-1,9$ & 5,03 & $4,3-5,8$ \\
\hline Acanthistius patachonicus & 8,5 & $7,0-10,1$ & 3,2 & $2,3-4,1$ & 3,3 & $2,1-4,7$ & 4,3 & $3,0-5,7$ & 4,98 & $4,2-5,6$ \\
\hline Seriorella porosa & 10,3 & $8,8-12,4$ & 2,4 & $1,7-3,2$ & 1,9 & $1,1-2,9$ & 3,9 & $2,6-5,2$ & 4,90 & $4,3-5,6$ \\
\hline Engraulis anchoita & 2,7 & $1,6-3,6$ & 3,9 & $3,0-5,0$ & 5,6 & $4,0-7,8$ & 7,7 & $6,0-9,7$ & 4,56 & $3,9-5,1$ \\
\hline Paralichtys patagonicus & & & & & 15,9 & $13,4-18,6$ & 1,8 & $1,0-2,8$ & 3,16 & $2,6-3,8$ \\
\hline Dipturus trachyderma & 0,4 & $0,1-0,8$ & 4,9 & $3,8-6,1$ & 5,2 & $3,6-6,7$ & 1,7 & $0,9-2,6$ & 3,02 & $2,5-3,5$ \\
\hline Eleginops maclovinus & 1,6 & $0,9-2,3$ & 3,0 & $2,1-4,0$ & 2,1 & $1,1-3,2$ & 2,8 & $1,8-3,9$ & 2,38 & $1,9-2,8$ \\
\hline Agonopsis chiloensis & 1,9 & $1,3-2,8$ & 0,7 & $0,3-1,2$ & 1,8 & $1,0-2,9$ & 4,7 & $3,4-6,2$ & 2,03 & $1,6-2,5$ \\
\hline Macruronus magellanicus & 5,0 & $3,9-6,2$ & 0,9 & $0,4-1,3$ & 0,2 & $0,0-0,7$ & 0,2 & $0,0-0,6$ & 1,83 & $1,4-2,3$ \\
\hline Odontesthes smitti & 1,3 & $0,7-1,9$ & 0,5 & $0,2-0,9$ & 1,2 & $0,5-2,0$ & 4,4 & $3,1-5,8$ & 1,59 & $1,2-2,0$ \\
\hline Squatina guggenheim & 0,8 & $0,3-1,4$ & 1,9 & $1,2-2,6$ & 1,8 & $0,9-2,7$ & 1,1 & $0,5-1,8$ & 1,39 & $1,1-1,7$ \\
\hline Pinguipes brasilianus & & & 2,9 & $2,0-4,1$ & 0,6 & $0,14-1,4$ & 0,7 & $0,2-1,4$ & 1,26 & $0,9-1,6$ \\
\hline Atlantoraja cyclophora & & & & & 4,5 & $3,1-5,9$ & 1,1 & $0,5-1,8$ & 1,00 & $0,7-1,3$ \\
\hline Myliobatis goodei & 0,5 & $0,0-1$ & 0,4 & $0,1-0,7$ & 1,3 & $0,6-2,3$ & 1,4 & $0,6-2,3$ & 0,77 & $0,5-1,1$ \\
\hline Psammobatis lentiginosa & & & 0,9 & $0,4-1,5$ & 1,8 & $0,9-3,0$ & 0,4 & $0,1-1,0$ & 0,70 & $0,5-0,9$ \\
\hline Polyprion americanus & 0,4 & $0,1-0,8$ & 0,9 & $0,5-1,4$ & 0,6 & $0,2-1,4$ & 0,5 & $0,1-1,0$ & 0,63 & $0,4-0,8$ \\
\hline Psammobatis rudis & 0,9 & $0,4-1,5$ & 0,1 & $0,0-0,3$ & 0,3 & $0,0-0,9$ & 0,1 & $0,0-0,4$ & 0,38 & $0,2-0,6$ \\
\hline Bathyraja macloviana & & & & & 0,1 & $0,0-0,3$ & 1,6 & $0,8-2,6$ & 0,32 & $0,2-0,5$ \\
\hline Brama brama & 0,3 & $0,1-0,6$ & 0,3 & $0,0-0,7$ & 0,5 & $0,1-1,1$ & 0,3 & $0,03-0,8$ & 0,32 & $0,2-0,5$ \\
\hline Myxine australis & & & & & 0,04 & $0,0-0,2$ & 1,4 & $0,7-2,4$ & 0,28 & $0,1-0,5$ \\
\hline Diplodus argenteus & 0,2 & $0,0-0,5$ & & & 0,1 & $0,0-0,5$ & 0,8 & $0,3-1,5$ & 0,24 & $0,1-0,4$ \\
\hline Iluocoetes fimbriatus & 0,04 & $0,0-0,2$ & & & 0,4 & $0,04-0,9$ & 0,1 & $0,0-0,5$ & 0,11 & $0,02-0,2$ \\
\hline Notorhynchus cepedianus & 0,1 & $0,0-0,3$ & 0,1 & $0,0-0,2$ & 0,1 & $0,0-0,5$ & 0,2 & $0,0-0,5$ & 0,10 & $0,02-0,2$ \\
\hline Bathyraja brachyurops & & & & & 0,5 & $0,1-1,1$ & 0,03 & $0,0-0,2$ & 0,10 & $0,01-0,2$ \\
\hline Triathalassothia argentina & & & & & 0,4 & $0,03-0,8$ & 0,1 & $0,0-0,4$ & 0,09 & $0,01-0,2$ \\
\hline Scomber japonicus & & & & & 0,3 & $0,0-0,7$ & 0,1 & $0,0-0,4$ & 0,07 & $0,01-0,2$ \\
\hline Notomyxine tridentiger & & & & & 0,2 & $0,0-0,6$ & 0,1 & $0,01-0,4$ & 0,06 & $0,01-0,2$ \\
\hline Leptonatus blainvilleanus & 0,1 & $0,0-0,4$ & & & 0,0 & $0,0-0,2$ & 0,03 & $0,0-0,2$ & 0,05 & $0,0-0,1$ \\
\hline Squalus mitsukurii & & & & & 0,2 & $0,0-0,5$ & 0,1 & $0,0-0,4$ & 0,05 & $0,0-0,1$ \\
\hline Dadyanos insignis & & & & & 0,2 & $0,0-0,6$ & & & 0,04 & $0,0-0,1$ \\
\hline Paranotothenia angustata & & & & & 0,1 & $0,0-0,3$ & 0,1 & $0,0-0,4$ & 0,03 & $0,0-0,1$ \\
\hline Austrolycus laticinctus & & & & & 0,1 & $0,0-0,5$ & 0,03 & $0,0-0,2$ & 0,03 & $0,0-0,1$ \\
\hline Micropogonias furnieri & 0,1 & $0,0-0,3$ & & & 0,04 & $0,0-0,2$ & & & 0,03 & $0,0-0,1$ \\
\hline Pomatomus saltatrix & 0,04 & $0,0-0,2$ & & & 0,1 & $0,0-0,4$ & & & 0,03 & $0,0-0,1$ \\
\hline Prionace glauca & 0,02 & $0,0-0,1$ & & & 0,04 & $0,0-0,2$ & 0,1 & $0,0-0,3$ & 0,03 & $0,0-0,1$ \\
\hline Sarda sarda & & & & & 0,1 & $0,0-0,4$ & & & 0,02 & $0,0-0,1$ \\
\hline Allocyttus verrucosus & & & & & 0,04 & $0,0-0,3$ & & & 0,01 & $0,0-0,04$ \\
\hline Notothenia magellanica & & & & & 0,04 & $0,0-0,3$ & & & 0,01 & $0,0-0,04$ \\
\hline Sprattus fueguensis & & & & & 0,04 & $0,0-0,3$ & & & 0,01 & $0,0-0,04$ \\
\hline
\end{tabular}


Tabla 5

Frecuencia de ocurrencia de las especies de peces capturadas incidentalmente en la pesquería de langostino patagónico por la flota congeladora tangonera por categoría de abundancia y destino, Do (dominante), Ab (abundante), Co (común), Ra (rara), En (encajonada), P Enc, (parcialmente encajonada) y De (descarte)

Frequency of occurrence of species in the bycatch of the Patagonian shrimp fishery, by class of abundance and destiny. Do (dominant), Ab (abundant), Co (common), Ra (rare), En (landing), P Enc, (partially landing) and De (discard)

\begin{tabular}{|c|c|c|c|c|c|c|c|c|}
\hline \multirow[b]{3}{*}{ Especie } & \multicolumn{8}{|c|}{$2003,2004,2006,2007$} \\
\hline & \multicolumn{4}{|c|}{ Abundancia } & \multicolumn{3}{|c|}{ Destino } & \multirow[b]{2}{*}{ Registros } \\
\hline & Do & $\mathrm{Ab}$ & Co & $\mathrm{Ra}$ & En & P Enc & De & \\
\hline Merluccius hubbsi & 29,95 & 39,00 & 18,44 & 12,61 & 0,62 & 17,87 & 81,36 & 15588 \\
\hline Patagonotothen ramsayi & 0,02 & 0,03 & 0,51 & 99,44 & 0,01 & 0,05 & 99,85 & 11661 \\
\hline Genypterus blacodes & 0,03 & 0,06 & 0,31 & 99,60 & 0,29 & 4,92 & 94,56 & 11502 \\
\hline Dipturus chilensis & 0,01 & 0,01 & 0,16 & 99,82 & 0,02 & & 99,82 & 10062 \\
\hline Psammobatis normani & 0,03 & 0,02 & 0,17 & 99,78 & & & 99,99 & 8954 \\
\hline Discopyge tschudii & 0,03 & & 0,22 & 99,76 & & & 99,97 & 7746 \\
\hline Stromateus brasiliensis & & 0,03 & 0,07 & 99,90 & & 0,06 & 99,87 & 6694 \\
\hline Sympterygia bonapartii & 0,04 & 0,02 & 0,09 & 99,86 & 0,02 & 0,04 & 99,77 & 5711 \\
\hline Schroederichthys bivius & 0,03 & 0,03 & 0,11 & 99,83 & & & 99,72 & 3625 \\
\hline Squalus acanthias & 0,03 & 0,06 & 0,15 & 99,76 & & & 99,91 & 3374 \\
\hline Congiopodus peruvianus & & & & 100 & 0,03 & 0,10 & 99,74 & 3112 \\
\hline Callorhinchus callorhynchus & 0,07 & & 0,63 & 99,30 & 0,55 & 1,18 & 98,27 & 2712 \\
\hline Xystreurys rasile & & 0,04 & 0,59 & 99,37 & 0,76 & 7,15 & 92,09 & 2377 \\
\hline Paralichtys isosceles & 0,14 & 0,05 & 1,29 & 98,52 & 0,53 & 2,30 & 97,17 & 2088 \\
\hline Cottoperca gobio & & & 0,05 & 99,95 & 0,30 & & 99,41 & 2025 \\
\hline Raneya brasiliensis & 0,10 & & 0,21 & 99,69 & & & 100 & 1932 \\
\hline Nemadactylus bergi & 0,79 & 4,05 & 10,22 & 84,94 & & & 100 & 1899 \\
\hline Genypterus brasiliensis & 0,06 & 0,26 & 0,06 & 99,61 & 0,51 & 1,41 & 98,01 & 1559 \\
\hline Sebastes oculatus & 0,08 & & 0,24 & 99,68 & 0,40 & 0,40 & 99,03 & 1235 \\
\hline Mustelus schmitti & 0,08 & & & 99,92 & 0,08 & & 99,84 & 1217 \\
\hline Percophis brasiliensis & 0,08 & & & 99,92 & & & 99,67 & 1207 \\
\hline Salilota australis & 0,09 & & 0,36 & 99,54 & 0,36 & 0,18 & 99,09 & 1099 \\
\hline Parona signata & & 0,09 & 0,65 & 99,26 & 2,86 & 3,51 & 93,63 & 1084 \\
\hline Pseudopercis semifasciata & 0,12 & & 0,12 & 99,77 & 7,62 & 3,17 & 88,98 & 853 \\
\hline Galeorhinus galeus & & & & 100 & 0,13 & 0,13 & 99,75 & 792 \\
\hline Acanthistius patachonicus & & 0,51 & 0,64 & 98,85 & 2,68 & 1,28 & 96,05 & 784 \\
\hline Seriorella porosa & 0,13 & 0,78 & 3,63 & 95,46 & 5,84 & 10,38 & 83,53 & 771 \\
\hline Engraulis anchoita & 1,11 & 1,53 & 5,29 & 92,06 & 0,14 & 5,15 & 94,71 & 718 \\
\hline Paralichtys patagonicus & & & & 100 & 0,40 & 5,43 & 94,16 & 497 \\
\hline Dipturus trachyderma & & & & 100 & & & 98,11 & 476 \\
\hline Eleginops maclovinus & & 0,27 & 0,27 & 99,47 & 13,07 & 2,93 & 84,00 & 375 \\
\hline Agonopsis chiloensis & 0,31 & & & 99,69 & & & 100 & 319 \\
\hline Macruronus magellanicus & & & & 100 & & & 99,65 & 288 \\
\hline Odonthestes smitti & 0,80 & 3,98 & 13,94 & 81,27 & 3,19 & 19,92 & 76,89 & 251 \\
\hline Squatina guggenheim & & & & 100 & & & 100 & 219 \\
\hline Pinguipes brasilianus & & & & 100 & & & 100 & 198 \\
\hline Atlantoraja cyclophora & & & & 100 & & & 100 & 158 \\
\hline Myliobatis goodei & & & & 100 & & & 100 & 121 \\
\hline Psammobatis lentiginosa & & & & 100 & & & 100 & 110 \\
\hline Polyprion americanus & & & & 100 & 19,19 & 2,02 & 78,79 & 99 \\
\hline Psammobatis rudis & & & & 100 & & & 100 & 60 \\
\hline Bathyraja macloviana & & & & 100 & 3,92 & & 96,08 & 51 \\
\hline Brama brama & & & & 100 & 37,25 & 1,96 & 60,78 & 51 \\
\hline Myxine australis & & & & 100 & & & 100 & 44 \\
\hline Diplodus argenteus & & & & 100 & 10,81 & & 89,19 & 37 \\
\hline Iluocoetes fimbriatus & & & & 100 & & & 100 & 17 \\
\hline Notorhynchus cepedianus & & & & 100 & & & 100 & 16 \\
\hline Bathyraja brachyurops & & & & 100 & & & 100 & 15 \\
\hline Ramnogaster arcuata & & 7,14 & 14,29 & 78,57 & & & 100 & 14 \\
\hline Triathalassothia argentina & & & & 100 & & & 100 & 14 \\
\hline Scomber japonicus & & & & 100 & 45,45 & & 54,55 & 11 \\
\hline Notomyxine tridentiger & & & & 100 & & & 100 & 10 \\
\hline Leptonotus blainvilleanus & & & & 100 & & & 100 & 8 \\
\hline Squalus mitsukurii & & & & 100 & & & 100 & 8 \\
\hline Dadyanos insignis & & & & 100 & & & 100 & 6 \\
\hline Paranotothenia angustata & & & & 100 & & & 100 & 5 \\
\hline Austrolycus laticinctus & & & & 100 & & & 100 & 4 \\
\hline Micropogonias furnieri & & & & 100 & & & 100 & 4 \\
\hline Pomatomus saltatrix & & & & 100 & & & 100 & 4 \\
\hline Prionace glauca & & & & 100 & 25,00 & & 75,00 & 4 \\
\hline Sarda sarda & & & & 100 & & & 100 & 3 \\
\hline Allocyttus verrucosus & & & & 100 & & & 100 & 1 \\
\hline Notothenia magellanica & & & & 100 & & & 100 & 1 \\
\hline Sprattus fuegensis & & & & 100 & & & 100 & 1 \\
\hline
\end{tabular}


El 40,6\% de las especies fue capturado con una FO menor al $1 \%$. Las especies raras capturadas incidentalmente pueden reflejar su condición natural dentro del ecosistema o la ineficiencia de los equipos de pesca para capturarlas. Sin embargo, la significancia ecológica de las especies raras y el impacto de las capturas sobre las mismas es difícil de evaluar, en general, debido al escaso conocimiento de ellas. Las especies raras tienden a ser impredecibles lo que dificulta el diseño de estrategias de manejo para protegerlas (Hall 1996, Hall et al. 2000).

Con excepción de $M$. hubbsi, las especies de peces fueron ocasionalmente consignadas como dominantes, abundantes o comunes en las capturas. Pese a ser eventos poco frecuentes, su impacto puede ser importante si el evento es catastrófico (Hall et al. 2000).

El manejo de esta pesquería ha priorizado el control de la captura incidental de merluza, lo cual es razonable dada su magnitud y la importancia comercial de este recurso. No obstante, es esencial determinar qué especies son más vulnerables al impacto de la captura para focalizar acciones adicionales de mitigación específicas, ya que la reducción de toda la captura incidental es difícil (Hall 1996).

Mas allá de aportar herramientas para los esquemas de manejo en la pesquería de langostino, el presente trabajo contribuye al conocimiento de la ictiofauna del golfo San Jorge y aguas contiguas.

En el Mar Argentino fueron registradas 39 especies de tiburones (Menni \& Lucifora 2007). N. cepedianus, P. glauca, S. bivius, M. schmitti, G. galeus, S. acanthias, $S$. mitsukurii y $S$. guggenheim fueron capturados en la pesquería de langostino.

Fueron registradas 34 especies de batoideos en el Mar Argentino (Cousseau et al. 2007); 11 especies integran la captura incidental de la pesquería de langostino patagónico: D. tschudii, A. cyclophora, D chilensis, $D$. trachyderma, B. brachyurops, B. macloviana, P. normani, $P$. lentiginosa, $P$. rudis, $S$. bonapartii y $M$. goodei. El registro de A. cyclophora en aguas del golfo San Jorge amplía su distribución respecto de lo descripto por Cousseau et al. (2007). Existen dos ejemplares en la colección ictiológica de la Facultad de Ciencias Naturales de la Universidad Nacional de la Patagonia (UNPSJB ICT/2005/22 y UNPSJB ICT/2005/27).

Se identificaron siete especies de clupeidos para el Mar Argentino (Cousseau et al. 2004), dos se observaron en las capturas de la pesquería de langostino: $S$. fueguensis y B. aurea. La presencia de B. aurea en aguas del golfo San Jorge amplía su distribución respecto de lo descripto por Cousseau \& Perrota (2000).

Sólo dos especies de espáridos son citados para el Mar Argentino (Cousseau et al. 2004): D. argenteus y $P$. pagrus. La presencia de $D$. argenteus y $P$. pagrus en aguas del golfo San Jorge amplía su distribución respecto de lo descripto por Galván et al. (2005).

Siete especies de sciénidos fueron registradas para el Mar Argentino (Cousseau et al. 2004). Sólo M. furnieri fue capturada en la pesquería de langostino. La presencia de esta especie en aguas del golfo San Jorge amplía su distribución según lo descripto por Cousseau \& Perrota (2000).

La familia Scombridae está representada por siete especies en el Mar Argentino (Cousseau et al. 2004). S. japonicus y $S$. sarda fueron capturadas en la pesquería de langostino. La presencia de $S$. sarda en aguas del golfo San Jorge amplía su distribución respecto de lo descripto por Cousseau \& Perrota (2000).

Por último, la familia Paralichthydae está representada por cinco especies en el Mar Argentino; fueron capturadas $X$. rasile, $P$. isosceles y $P$. patagonicus. La presencia de $P$. patagonicus amplía su distribución según lo descripto por Cousseau \& Perrota (2000). Existen dos ejemplares en la colección ictiológica UNPSJB (UNPSJB ICT/2005/ 45 y UNPSJB ICT/2005/46).

El trabajo fue realizado gracias a la información proveniente del Programa de Observadores a Bordo de la Provincia del Chubut, y enfatiza la utilidad de los programas de observadores para evaluar el problema del descarte (Kelleher 2008) y aportar información sobre la biodiversidad y distribución de las especies que componen los ecosistemas marinos (Fennessy et al. 1994).

\section{Agradecimientos}

Este estudio fue posible gracias al Programa de Observadores coordinado por la Secretaría de Pesca de la Provincia del Chubut. Agradecemos el invalorable trabajo de los observadores a bordo, en especial Fabián García, Marcelo Schmidth, Gonzalo Quiroga, Rubén Cambursano, Juan José Romero, Gabriel Alonso y Leonardo Jerez, y también a los miembros del Programa por su colaboración: Luis Mendia, Matías Soutric, Mauricio Gallardo Gallardo, Néstor Santibáñez y Graciela Sarsa. Un agradecimiento especial al Dr. Atila Gosztonyi por su asesoramiento en la identificación taxonómica y a la Dra. Ana Parma por sus aportes en la edición de este manuscrito. Las sugerencias de los evaluadores han permitido mejorar sustancialmente el manuscrito, a ellos también un agradecimiento especial. 


\section{Literatura citada}

Aubone A, SI Bezzi, G Cañete, R Castrucci, C Dato, G Irusta, A Madirolas, M Pérez, M Renzi, B Santos, M Simonazzi \& MF Villarino. 2004. Evaluación y sugerencias de manejo del recurso merluza (Merluccius hubbsi). La situación hasta 1999. En: Sánchez RP \& SI Bezzi (eds). El Mar Argentino y sus recursos pesqueros. Tomo 4 . Los peces marinos de interés pesquero. Caracterización biológica y evaluación del estado de explotación, pp. 207-235. Instituto de Investigación y Desarrollo Pesquero, Mar del Plata.

Bezzi SI, M Renzi, G Hirsuta, B Santos, LS Tringali, MD Ehrlich, F Sánchez, SB García de la Rosa, M Simonazzi \& MF Villarino. 2004. Caracterización biológica y pesquera de la merluza (Merluccius hubbsi). En: Sánchez RP \& SI Bezzi (eds). El Mar Argentino y sus recursos pesqueros. Tomo 4 . Los peces marinos de interés pesquero. Caracterización biológica y evaluación del estado de explotación, pp. 157-205. Instituto de Investigación y Desarrollo Pesquero, Mar del Plata.

Cedrola PV, AM González \& AD Pettovello. 2004. Bycatch of skates (Elasmobranchii: Arhynchobatidae, Rajidae) in the Patagonian red shrimp fishery. Fisheries Research 71 : 141-150.

Cousseau MB \& RG Perrota. 2000. Peces marinos de Argentina. Biología, distribución, pesca, 167 pp. Instituto de Investigación y Desarrollo Pesquero, Mar del Plata.

Cousseau MB, AE Gosztonyi, I Elías \& ME Ré. 2004. Estado actual del conocimiento de los peces de la plataforma continental argentina y adyacencias. En: Sánchez RP \& SI Bezzi (eds). El Mar Argentino y sus recursos pesqueros. Tomo 4. Los peces marinos de interés pesquero. Caracterización biológica y evaluación del estado de explotación, pp. 17-38. Instituto de Investigación y Desarrollo Pesquero, Mar del Plata.

Cousseau MB, DE Figueroa, JM Díaz de Astarloa, E Mabragaña \& LO Lucifora. 2007. Raya, chuchos y otros batoideos del Atlántico Sudoccidental $\left(34^{\circ} \mathrm{S}-55^{\circ} \mathrm{S}\right), 102$ pp. Instituto de Investigación y Desarrollo Pesquero, Mar del Plata.

Crawley M. 2007. The R Book, 942 pp. John Wiley \& Sons, New York.

Eschmeyer WN. 2007. Catalog of fishes. Online Version, Updated 9 September 2009. California Academy of Sciences. [en línea] <http://research.calacademy.org/ research/ichthyology/catalog/fishcatmain.asp>
FAO. 2003. La ordenación pesquera. 2. El Enfoque de ecosistema en la pesca. FAO Orientaciones Técnicas para la Pesca Responsable 4(Suppl. 2): 1-113.

Fennessy ST, C Villacastin \& JG Field. 1994. Distribution and seasonality of ichthyofauna associated with commercial prawn trawl catches on the Tugela Bank of Natal, South Africa. Fisheries Research 20: 263-282.

Galvan D, L Venerus, A Irigoyen, AM Parma \& AE Gosztonyi. 2005. Extension of the distributional range of the silver porgy, Diplodus argenteus (Valenciennes 1830), and the red porgy, Pagrus pagrus (Linnaeus 1758) (Sparidae) in northern Patagonia, south-western Atlantic. Journal of Applied Ichthyology 21: 444-447.

Hall MA. 1996. On bycatches. Reviews in Fish Biology and Fisheries 6: 319-352.

Hall MA, DL Alverson \& KI Metuzals. 2000. Bycatch: Problems and solutions. Marine Pollution Bulletin 41(16): 204-219.

Kelleher K. 2008. Descartes en la pesca de captura marina mundial. FAO Documento Técnico de Pesca 470: 1-147.

Menni RC \& A Gosztonyi. 1982. Benthic and semidemersal fish association in the Argentine Sea. Studies on Neotropical Fauna and Environment 17: 1-29.

Menni RC \& LO Lucifora. 2007. Condrictios de la Argentina y Uruguay. Lista de trabajo. ProBiota, FCNyM, Universidad Nacional de La Plata, Serie Técnica-Didáctica, La Plata, Argentina 11: 1-15. [en línea] < http://aquacomm.fcla.edu/ 1670/1/11-_Lista_Condrictios.pdf >

Pettovello A. 1999. Bycatch in the Patagonian red shrimp (Pleoticus muelleri) fishery. Marine and Freshwater Research 50: 123-127.

Roux A, R Piñero \& J de la Garza. 2007. Guía para la identificación de la fauna asociada a la pesquería de langostino patagónico, 32 pp. Instituto de Investigación y Desarrollo Pesquero, Mar del Plata.

Stobuzki IC, MJ Miller, P Jones \& JP Salini. 2001. Bycatch diversity and variation in a tropical Australian penaeid fishery; the implications for monitoring. Fisheries Research 53: $283-301$.

Vianna M \& T Almeida. 2005. Bony fish bycatch in the Southern Brazil pink shrimp (Farfantepenaeus brasiliensis and $F$. paulensis) Fishery. Brazilian Archives of Biology and Technology 48(4): 611-623. 